\title{
WATTS COHOMOLOGY OF FIELD EXTENSIONS ${ }^{1}$
}

\section{NEWCOMB GREENLEAF}

Let $R$ be a commutative ring and $A$ a commutative $R$-algebra. In [4] Watts defined a cohomology theory, $H_{K}^{n}(A)$, which yields the Cech cohomology of the compact Hausdorff space $X$ in the case when $R=R$.and $A=C(X)$, the ring of continuous real valued functions on $X$. The definition of $H_{R}^{n}(A)$ was in terms of a specific complex derived from the "additive Amitsur complex." The question of the possible functorial significance of this cohomology theory was raised. As a step in this direction we compute here the Watts cohomology $H_{K}^{n}(L)$, where $K$ is a field and $L$ is an arbitrary extension field of $K$.

We recall the definition of $H_{K}^{n}(L)$. The complex $F_{K}^{*}(L)$ is the additive Amitsur complex [3] with a dimension shift of $1: F_{K}^{n}(L)$ is the $n+1$-fold tensor product of $L$ over $K$, and the coboundary map $d^{n}: F_{K}^{n}(L) \rightarrow F_{K}^{n+1}(L)$ is given by $d^{n}\left(x_{0} \otimes \cdots \otimes x_{n}\right)=\sum_{i=0}^{n+1}(-1)^{i} x_{0} \otimes \cdots \otimes x_{i-1} \otimes 1 \otimes x_{i} \otimes \cdots \otimes x_{n}$.

The homology of this complex is easily found.

Proposition 1. The complex $F_{K}^{*}(L)$ has zero homology except in dimension zero, where $H^{0}\left(F_{K}^{*}(L)\right) \cong K$.

Proof. It is known [3, Lemma 4.1] that the complex $0 \rightarrow K \rightarrow F_{K}^{0}(L)$ $\rightarrow F_{K}^{1}(L) \cdots$ is acyclic.

Let $\mu_{n}: F_{K}^{n}(L) \rightarrow L$ by $\mu_{n}\left(x_{0} \otimes \cdots \otimes x_{n}\right)=x_{0} \cdots x_{n}$. The subcomplex $N_{\boldsymbol{K}}^{\cdot}(L)$ is given by

$N_{K}^{n}(L)=\left\{x \in F_{K}^{n}(L) \mid \exists y \in F_{K}^{n}(L) \quad\right.$ with $\quad \mu_{n}(y) \neq 0 \quad$ and $\left.\quad x y=0\right\}$

(the definition is simplified here by the fact that $L$ is a field). Note that $N_{K}^{n}(L) \subseteq$ ker $\mu$. The Watts cohomology $H_{K}^{*}(L)$ is then defined to be the homology of the quotient complex $C_{K}^{*}(L)=F_{K}^{*}(L) / N_{K}^{*}(L)$. Let $\pi_{n}: F_{K}^{n}(L) \rightarrow C_{K}^{n}(L)$ denote the standard map.

Let $L_{\mathrm{s}}$ be the separable closure of $K$ in $L$. We shall prove the following

Theorem. The complexes $C_{K}^{\cdot}(L)$ and $F_{L_{s}}^{\cdot}(L)$ are canonically isomorphic.

Received by the editors March 25, 1968.

${ }^{1}$ This research was partially supported by National Science Foundation Grant GP-7436. 
The following corollary is then immediate by Proposition 1.

Corollary. Watts cohomology for field extensions is given by $H_{K}^{0}(L) \cong L_{s}$ and $H_{K}^{n}(L)=0$ for $n>0$.

We now establish the theorem.

Proposition 2. Let $L$ be separable algebraic over $K$, and let $x \in F_{K}^{n}(L)$ with $\mu_{n}(x)=0$. Then there is $a y \in F_{K}^{n}(L)$ with $\mu_{n}(y) \neq 0$ and $x y=0$.

Proof. It suffices to consider the case where $L$ is a finite extension of $K$. But then $F_{R}^{n}(L)$ is a semisimple ring and every ideal is a direct factor. Hence $F_{K}^{n}(L) \cong L^{\prime} \times \operatorname{ker}\left(\mu_{n}\right)$, where $L^{\prime}$ is a field and $\mu_{n}$ maps $L^{\prime}$ isomorphically onto $L$.

CoRollaRy. If $L$ is separable algebraic over $K, N_{K}^{n}(L)=\operatorname{ker}\left(\mu_{n}\right)$, and there is a canonical isomorphism $\beta_{n}: C_{K}^{n} \rightarrow L$ such that $\beta_{n} \circ \pi_{n}=\mu_{n}$.

Proposition 3. Let $K$ be separably closed in $L$ (i.e. $L_{s}=K$ ) and let $A$ be a commutative $K$-algebra in which every zero divisor is nilpotent. Then every zero divisor in $A \otimes_{K} L$ is nilpotent.

Proof. We may work within a finitely generated subalgebra of $A$, and hence assume $A$ is Noetherian. Let $N$ be the ideal of nilpotents in $A$. Then (0) is a primary ideal in $A$ and $N$ is its associated prime. It then follows [1, Chapter IV, \$2.6, Theorem 2] (with $E=A, F=B$ $\left.=A \otimes_{K} L\right)$ that the associated prime ideals of (0) in $A \otimes_{K} L$ coincide with the associated prime ideals of the ideal $N \otimes_{K} L$ in $A \otimes_{K} L$. But by $\left[2\right.$, Chapter IV, Theorem 24] every zero-divisor in $A / N \otimes_{K} L$ is nilpotent and hence $N \otimes_{K} L$ is a primary ideal.

CoROLlaRy. If $K$ is separably closed in $L$, then every zero divisor in $F_{K}^{n}(L)$ is nilpotent, for all $n$, and $F_{K}^{n}(L)=C_{K}^{n}(L)$.

Now let $\theta_{n}: F_{K}^{n}(L) \rightarrow F_{L_{s}}^{n}(L)$ by $\theta_{n}\left(x_{0} \otimes \cdots \otimes x_{n}\right)=x_{0} \otimes \cdots \otimes x_{n}$. It is clear that $\theta_{n}$ is surjective and that $\theta=\left\{\theta_{n}\right\}$ is a map of complexes, $\theta: F_{K}^{\cdot}(L) \rightarrow F_{L_{s}}^{*}(L)$. Let $x \in N_{K}^{n}(L)$, with $y$ satisfying $x y=0, \mu_{n}(y) \neq 0$. Then $\theta_{n}(x) \theta_{n}(y)=0$ and $\mu_{n}\left(\theta_{n}(y)\right)=\mu_{n}(y) \neq 0$, so $\theta_{n}(x) \in N_{L_{s}}^{n}(L)=(0)$. Hence $\theta$ induces a surjective map of complexes $\tau: C_{K}(L) \rightarrow C_{L_{s}}(L)$ $=F_{L_{a}}(L)$. To complete the proof of the theorem we will construct an inverse to $\tau$.

Fix an integer $n$, and let $A$ denote the ring $F_{k}^{n}(L), B$ the subring $F_{K}^{n}\left(L_{s}\right)$. If $M$ is an $A$-module and $\rho: A \rightarrow M$ an $A$-linear map, then $\operatorname{ker}(\rho) \supset A\left(\left.\operatorname{ker} \rho\right|_{B}\right)$ and hence $\rho$ factors as $A=A \otimes_{B} B \rightarrow A \otimes_{B} \rho(B)$ $\rightarrow M$. We apply this method to the two maps $\theta_{n}: A \rightarrow F_{L_{s}}^{n}(L)$, and $\pi_{n}: A \rightarrow C_{K}^{n}(L)$. 
Proposition 4. The induced map $A \otimes_{B} \theta_{n}(B)=F_{K}^{n}(L) \otimes_{F_{K}^{n}\left(L_{s}\right)} L_{s}$ $\rightarrow F_{L_{s}}^{n}(L)$ is an isomorphism.

Proof. First note that $\theta_{n}(B)$ is canonically isomorphic to $L_{s}$. We construct an inverse. Let $\omega: L \times \cdots \times L \rightarrow A \otimes_{B} \theta_{n}(B)$ by $\omega\left(x_{0}, \cdots, x_{n}\right)$ $=x_{0} \otimes \cdots \otimes x_{n} \otimes 1 . \quad$ If $\quad y \in L_{s}, \quad x_{0} \otimes \cdots \otimes y x_{i} \otimes \cdots \otimes x_{n} \otimes 1$ $=x_{0} \otimes \cdots \otimes x_{i} \otimes \cdots \otimes x_{n} \otimes y$. Hence $\omega$ is $L_{s}$-multilinear and induces $F_{L_{s}}^{n}(L) \rightarrow A \otimes_{B} \theta_{n}(B)$.

Consider the following diagram:

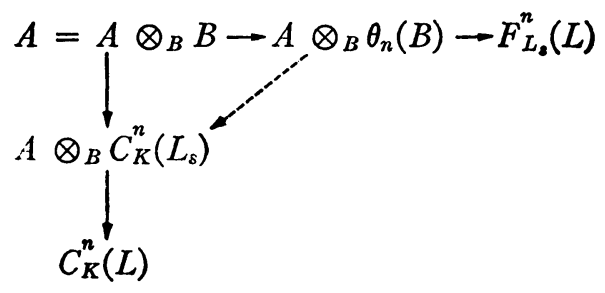

Using Propositions 3 and $4, \theta_{n}(B) \cong L_{8} \cong C_{K}^{n}\left(L_{8}\right)$ and hence we obtain, by Proposition 4,

$$
F_{L_{s}}^{n}(L) \rightarrow A \otimes_{B} \theta_{n}(B) \rightarrow A \otimes_{B} C_{K}^{n}\left(L_{s}\right) \rightarrow C_{K}^{n}(L),
$$

and the composite map is easily seen to be inverse to $\tau_{n}$.

The author would like to thank the referee for supplying an elegant alternative to a clumsy induction.

\section{REFERENCES}

1. N. Bourbaki, Algèbre commutative, Chapter IV, Hermann, Paris, 1961.

2. N. Jacobson, Lectures in abstract algebra, Vol. III, Van Nostrand, Princeton, N.J., 1964.

3. A. Rosenburg and D. Zelinski, On Amitsur's complex, Trans. Amer. Math. Soc. 97 (1960), 327-356.

4. C. Watts, Alexander-Spanier cohomology and rings of continuous functions, Proc. Nat. Acad. Sci. U.S.A. 54 (1965), 1027-1028.

UNIVERSITY OF ROCHESTER 